\title{
Morphology and Chemical Composition of PM10 by SEM-EDS
}

\author{
R. Ramirez-Leal, M. Valle-Martinez and M. Cruz-Campas
}

State University of Sonora, Ley Federal del Trabajo Final s/n, Col. Apolo Hermosillo, Sonora, Mexico

The physical-chemical, morphological and dimensional determinations of the complex mixture of organic and inorganic particulates are one of the major aspects for their characterization and for identification of emission sources that contribute to particulate air concentrations. (2)

The World Health Organization (WHO, 2003) suggests that besides physical aspects such as particle number, size, or surface, the chemical composition of particles is likely to play a crucial role with regards the health implications of particulate matter. Various health effects of Particulate Matter, from less serious to very serious ones, are associated with its specific chemical and physical components; and are an important determining factor in their toxicological effect. (4)

Particulate matter of aerodynamic diameter less than $10 \mu \mathrm{m}$ (PM10), has been found to be associated with urban health problems. Many epidemiological studies show that atmospheric aerosols may produce adverse health effects, with recent studies revealing that coarser atmospheric particles are more related to respiratory diseases, whereas the finest particles seem to affect the cardio-vascular system. (3)

The morphologic and chemical characterizations of particulate matter PM10 are suitable tools in the identification of the pollutant source: natural or anthropogenic. Its morphology can be affected by factors such as the process of formation of the particles, in addition to the physical and chemical properties of the superficial layers of the particles. (1)

This work is based on samplings PM10 collected in three atmospheric monitoring stations of the Air Quality Improvement Municipal Program for the City of Hermosillo, Sonora, Mexico.

The aim of this work is identification and characterization (morphology and chemical composition) of particles present in samples of PM10 as part of an air quality study by SEM-EDS

To determine the chemical composition and morphology of particles at the individual level, a scanning electron microscope (SEM) JEOL JSM-5800LV coupled with an energy dispersed spectrometry (EDS) microanalysis EDAX DX4 was used, with a lower detection limit per element of less than $0.1 \%$. For this study we use quartz filters; was placed a filter section in a test tube containing Isopropyl alcohol. Then, an aliquot of the suspension was placed over a sample holder and into the SEM.

The City of Hermosillo is located between latitude $20^{\circ} 01^{\prime} 00^{\prime \prime}$ and $20^{\circ} 08^{\prime} 30^{\prime \prime}$ north latitude and between the meridian $110^{\circ} 54^{\prime} 30^{\prime \prime}$ and $111^{\circ} 01^{\prime} 00^{\prime \prime}$ west longitude at an altitude of $200 \mathrm{~m}$ above sea level; is the Sonora state's capital, located to the Center-West of the coastal plain, in northwestern mexico.

We obtained 132 images of particles PM10, were analyzed by SEM-EDS and then classified into 6 main groups, according to their chemical composition and morphology. Table 1.

The structure of particles can be diverse and they usually present a two dimensional aspect. It is often found that such particles have porous texture, irregular, spheroidal, crystals tabular, outlying edges and fracture lines. Fig. 1

Elements such as $\mathrm{Al}, \mathrm{Si}, \mathrm{Ca}, \mathrm{Fe}, \mathrm{K}$, and $\mathrm{Mg}$ are largely abundant, and are usually related with litophilic sources, While elements such as $\mathrm{Cl}$ are more frequently associated with incineration and combustion activities as well as derived from marine aerosols.

Given that there is relatively little knowledge on morphology, speciation and size of particles, caught on the PM10 filters it highlights the importance of individual characterization of atmospheric aerosol. This knowledge is valuable additional information, within air quality program, about the possible actions and 
potential effects that air pollutants, of natural or anthropogenic origins; may have on the population in the study area.

\section{References}

[2] 1.- J.J. Goldstein et al, Scanning Electrón Microscopy and X-ray Microanálisis, Plenum, New Cork, 1992.

[2] 2.-Ramirez-Leal,R., Esparza-Ponce,H and Duarte-Moller,A. 2007. Characterizatioin of inorganic atmospheric particles in air quality program with sem, tem and xas. Revista Mexicana de Fisica 53(3): 102-107.

[2] 3.- R. Ramirez-Leal, Microsc. Microanal. 15 (Suppl. 2) (2009) 1300-1301

[2] 4.- Hays, D.M., Fine, M.P., Geron, D.C., Kleeman, J.M., Gullett, K.B., 2005. Open burning of agricultural biomass: physical and chemical properties of particle-phase emissions. Atmospheric Environment 39 (36), 6747-6764

[2] 5.- Yunbo Zhai \& Zongmin Fu and Lafang Wang. 2012. Characteristic, composition, and sources of TSP investigated by HRTEM/EDS and ESEM/EDS. Environ Monit Assess (2012) 184:6693-6707


Fig. 1 Particle type. a)Marine aerosol; b)Litophilic source; c) Combustion source

Table 1. Classification main SEM-EDS of PM10

\begin{tabular}{|c|c|c|}
\hline GROUPS & SUB GROUPS & $\begin{array}{c}\text { Morphology and texture } \\
\text { of Particles }\end{array}$ \\
\hline Fe- rich & iron oxide & Irregular \\
\hline Si-rich & ilmenite (FeTiO3) & edges defined \\
\hline Ba- rich & $\begin{array}{c}\text { Oxide of silica-aluminium whit } \\
\text { trace Na-Ca }\end{array}$ & edges defined \\
\hline V-Ni- rich & barite (BaSO4) & irregular \\
\hline Pb- rich & vanadium-nickel & irregular and spheroidal \\
\hline Cl-Na- rich & lead oxide and sulphate & crystals tabular \\
\hline
\end{tabular}

